\title{
Moderate hypofractionated image-guided thoracic radiotherapy for locally advanced node-positive non-small cell lung cancer patients with very limited lung function: a case report
}

\author{
Farkhad Manapov, MD, Olarn Roengvoraphoj, MD, Minglun Li, MD, Chukwuka Eze, MD \\ Department of Radiation Oncology, Ludwig-Maximilian University of Munich, Germany
}

\begin{abstract}
Patients with locally advanced lung cancer and very limited pulmonary function (forced expiratory volume in 1 second [FEV1] $\leq$ $1 \mathrm{~L}$ ) have dismal prognosis and undergo palliative treatment or best supportive care. We describe two cases of locally advanced node-positive non-small cell lung cancer (NSCLC) patients with very limited lung function treated with induction chemotherapy and moderate hypofractionated image-guided radiotherapy (Hypo-IGRT). Hypo-IGRT was delivered to a total dose of 45 Gy to the primary tumor and involved lymph nodes. Planning was based on positron emission tomography-computed tomography (PET/ CT) and four-dimensional computed tomography (4D-CT). Internal target volume (IVV) was defined as the overlap of gross tumor volume delineated on 10 phases of 4D-CT. ITV to planning target volume margin was $5 \mathrm{~mm}$ in all directions. Both patients showed good clinical and radiological response. No relevant toxicity was documented. Hypo-IGRT is feasible treatment option in locally advanced node-positive NSCLC patients with very limited lung function (FEV1 $\leq 1 \mathrm{~L})$.
\end{abstract}

Keywords: Hypofractionated radiotherapy, Image-guided radiotherapy, NSCLC, Thoracic radiotherapy

\section{Introduction}

Locally advanced node-positive lung cancer patients often present with limited lung function due to chronic pulmonary comorbidities. Because conventional thoracic irradiation and chemoradiotherapy (CRT) are associated with significant pulmonary toxicity, these high-risk patients undergo palliative treatment or best supportive care alone [1].

Stereotactic body radiotherapy (SBRT) is an established treatment option in early-stage inoperable non-small cell lung cancer (NSCLC) [2]. SBRT was also shown to be feasible and safe in patients with limited lung function. Furthermore, several small trials have demonstrated that accelerated hypofractionated thoracic irradiation can be safely delivered in locally advanced lung cancer $[3,4]$. Nevertheless, these small trials included patients with adequate pulmonary function exclusively. There is no data on the feasibility of multimodality treatment in locally advanced node-positive patients with reduced lung function

Two high-risk patients with very limited lung function (forced expiratory volume in 1 second [FEV1] $\leq 1 \mathrm{~L}$ ) and locally advanced node-positive disease were treated with induction

Received 16 March 2017, Revised 05 May 2017, Accepted 29 May 2017.

Correspondence: Farkhad Manapov, MD, Department of Radiation Oncology, Ludwig-Maximilian University of Munich, Marchioninistraße 15, 81377 Munich, Germany. Tel: +49-89-4400-73770, Fax: +49-89-4400-76770, E-mail: Farkhad.Manapov@ med.uni-muenchen.de

(c) This is an Open Access article distributed under the terms of the Creative Commons Attribution Non-Commercial License (http://creativecommons.org/ licenses/by-nc/4.0/) which permits unrestricted non-commercial use, distribution, and reproduction in any medium, provided the original work is properly cited.

www.e-roj.org 
chemotherapy followed by hypofractionated image-guided radiotherapy (Hypo-IGRT). In the following report, we describe these cases with follow-up of 9 months.

\section{Case Report}

\section{Patients}

A 72-year-old woman (current non-smoker) with the Eastern Cooperative Oncology Group (ECOG) performance status 2 presented with stage IIIA NSCLC. She had chronic obstructive pulmonary disease (COPD) Global Initiative for Chronic Obstructive Lung Disease (GOLD) III at baseline. Tumor-related symptoms included worsening of dyspnea. The histology was an adenocarcinoma of the lung, epidermal growth factor receptor (EGFR) wild-type without anaplastic lymphoma kinase (ALK)-translocation. 18F-FDG/PET-CT (positron emission tomography with 2-deoxy-2-[fluorine-18]-fluoro-D-glucose integrated with computed tomography) in treatment position showed the primary tumor with pleural contact in the right upper lobe and positive lymph nodes in the ipsilateral hilum and mediastinum. The pulmonary function tests revealed a vital capacity (VC) of $2.02 \mathrm{~L}$, FEV1 of $1.0 \mathrm{~L}$ (60\% predicted) and an estimated DLCO (diffusion capacity for carbon monoxide) of $35 \%$ of the norm. Induction chemotherapy constituting cisplatin $80 \mathrm{mg} / \mathrm{m}^{2}$ IV (intravenous) day 1 and pemetrexed $500 \mathrm{mg} / \mathrm{m}^{2}$ IV day 1 (every 3 weeks) was initially administered, leading to stable disease. Two and a half months after the last cycle of chemotherapy, Hypo-IGRT was delivered.

A 62-year-old man (current non-smoker) with ECOG performance status 2 presented with stage IIIA NSCLC. He had COPD GOLD IV at baseline and was on supplemental oxygen. Tumor-related symptoms included pain in the parasternal region and worsening of dyspnea. The histology revealed an adenocarcinoma of the lung, EGFR wild-type without ALKtranslocation. FDG/PET-CT scan in treatment position showed the primary tumor with pleural contact in the left upper lobe and positive lymph nodes in the ipsilateral mediastinum. The pulmonary function tests revealed a VC of $2.66 \mathrm{~L}$, FEV1 of 0.84 $\mathrm{L}(56 \%$ predicted) and DLCO was not measurable. Induction chemotherapy was also administered analog the abovementioned case, leading to stable disease. Two months after the last cycle of chemotherapy, Hypo-IGRT was delivered.

\section{Hypo-IGRT}

Hypo-IGRT was performed following chemotherapy. Patients underwent PET-CT and four-dimensional computed tomography (4D-CT) (10 sequential scans in all respiratory phases) in the treatment position (vacuum pillow and alphacradle). 4D-CT was matched with PET-CT for better definition of the target volume. The range of tumor motion was 1-4 $\mathrm{mm}$ in every dimensional space. Gross tumor volume (GTV) included primary tumor and involved lymph nodes (shortaxis $\geq 1 \mathrm{~cm}$ and/or PET-positive) post-induction chemotherapy. Internal target volume (ITV) was defined through the overlap of GTVs delineated on 10 phases of 4D-CT. clinical target volume was not generated in both cases, due to much reduced pulmonary reserve. Isotropic margin of $5 \mathrm{~mm}$ was added to ITV to generate the planning target volume (PTV). PTV was 255.5 and $314.28 \mathrm{~cm} 3$ in patient \#1 and \#2, respectively. Dose was calculated in $3 \mathrm{~mm}$ grid with Collapse-Cone algorithm (Fig. 1).

Total dose to PTV was $45 \mathrm{~Gy}$ in 15 fractions, 5 times a week and was prescribed per ICRU with corrections for lung inhomogeneity. Pulmonary dose-volume parameters were calculated with total lung volume minus PTV. Mean lung dose (MLD), averages of the ipsilateral and contralateral lung were 10.6 Gy, $14.45 \mathrm{~Gy}$, and $4.98 \mathrm{~Gy}$ in patient \# 1 and $9.4 \mathrm{~Gy}, 13.99$ $\mathrm{Gy}$, and $5.17 \mathrm{~Gy}$ in patient \#2, respectively. In the first patient, $V_{5}$ and $V_{10}$ of both lungs were $52 \%$ and $32 \%$, respectively. In the second patient, $V_{5}$ and $V_{10}$ were $48 \%$ and $29 \%$. $V_{20}$ (both lungs) was $19.1 \%$ and $15 \%$ in patients \#1 and \#2, respectively. Radiation was delivered by linear accelerator (Elekta Synergy, Stockholm, Sweden) using $15 \mathrm{MeV}$ 8-9 coplanar fixed photon beams. Image guidance was performed with kilovoltage-conebeam CT prior to each treatment. Toxicity monitoring was performed twice weekly to record any side effects.

\section{Treatment tolerability}

The first patient presented with grade 2 esophagitis (Common Terminology Criteria for Adverse Events v4.0) during last 4 days of treatment. Hypo-IGRT was not interrupted and the symptoms were resolved 5 days following irradiation. Lung function after treatment was at 1 month VC/FEV1/DLCO 1.87 L/0.88 L (53.6\% predicted)/27.4\%; at 3 months $1.73 \mathrm{~L} / 0.98$ $\mathrm{L}(59.8 \%$ predicted)/no data; and at 6 months $1.65 \mathrm{~L} / 0.88 \mathrm{~L}$ (55\% predicted) $/ 27.0 \%$, respectively. No symptoms relative to radiation pneumonitis (CT thorax 6 weeks and 3 months postradiation) and/or worsening of the baseline COPD-related symptoms were observed. The Patient achieved good partial response and she is progression-free after 9 months followup. Lung function at 9-month follow-up revealed VC/FEV1/ DLCO 1.61 L/0.98 L (59.9\% predicted)/22\%.

The second patient presented with an acute COPD exacerbation during the first week of Hypo-IGRT. Immediate hospitalization with application of systemic antibiotics and 

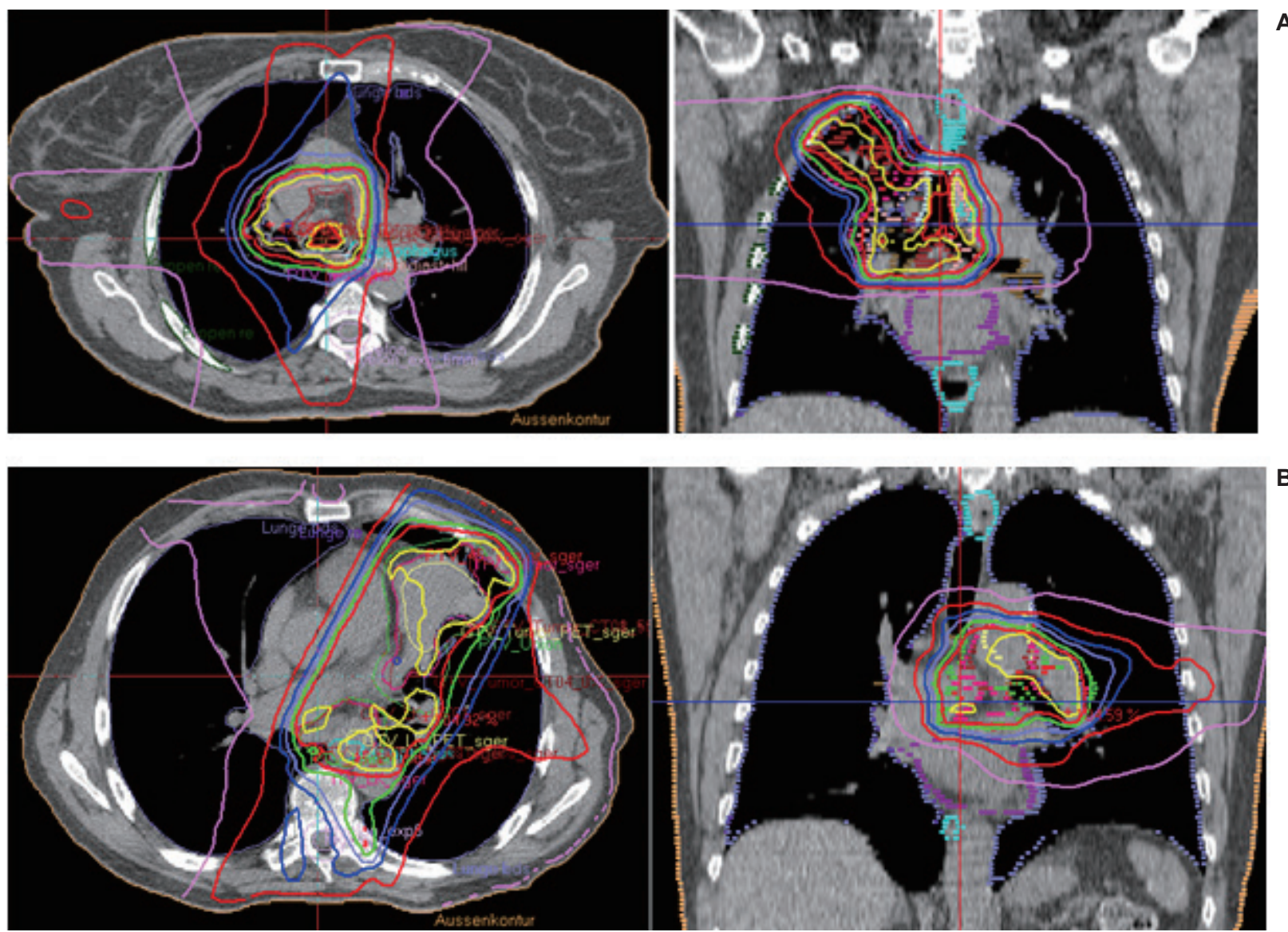

Fig. 1. Isodose distribution in selected planning computed tomography slices in patient \# 1 (A) and patient \#2 (B), respectively.

steroids was necessary. He developed grade 2 esophagitis at the end treatment, which was self-limiting after 3 weeks. Hypo-IGRT was interrupted for 3 days. Convalescence occurred 4 days following hospitalization. Lung function following treatment with bronchodilators, steroids, oxygen und pulmonary rehabilitation therapy was at 1 month VC/ FEV1/DLCO $2.22 \mathrm{~L} / 0.93 \mathrm{~L}$ (30.8\% of predicted)/not measurable and at 3 months with $2.16 \mathrm{~L} / 0.86 \mathrm{~L}$ (28.4\% predicted)/not measurable. Pulmonary function test at 6 months was refused by the patient. No symptoms of radiation pneumonitis on CT and/or worsening of the baseline COPD-related symptoms were observed. Partial response was achieved and remained stable, but distant progression after 6 months follow-up was documented and second-line chemotherapy was initiated.

\section{DISCUSSION}

The present case report demonstrates that moderate HypoIGRT can be considered as a feasible treatment in locally advanced NSCLC patients with very limited lung function.
An important requirement of our radiation treatment was an accurate transfer of SBRT technique for early-stage lung cancer to these high-risk node-positive patients. We have chosen a moderate dose prescription concept with overall duration of 3 weeks (15 fractions at 3 Gy per fraction once daily, 5 times a week) and corresponding biologically effective dose $10 \mathrm{~Gy}$ (BED $\alpha / \beta$-ratio 10 Gy assumed) of $58.5 \mathrm{~Gy}$ according to Amini et al. [3]. Local effectiveness of this moderate accelerated hypofractionated protocol was previously extensively described $[3,4]$. Important prerequisites referring to the treatment planning were also maximal accepted MLD and $V_{20}$ (both lungs) of $10 \mathrm{~Gy}$ and 20\% to significantly reduce the risk of symptomatic pneumonitis [5-7]. Both patients completed Hypo-IGRT with good partial response and complete regression of the tumor-related symptoms. Although we observed a mild decline of the pulmonary function parameters (Table 1) 3 to 6 months after Hypo-IGRT, no worsening of the baseline COPD-related symptoms and radiation pneumonitis were documented.

Chiang et al. [8] reported the effectiveness and feasibility of 
Table 1. Treated volumes and lung function parameters pre- and 3- (patient \#2) to 6-month (patient \# 1) post-Hypo-IGRT

\begin{tabular}{|c|c|c|c|c|c|c|c|c|c|c|}
\hline \multirow{2}{*}{ Patient no. } & \multirow{2}{*}{$\begin{array}{l}\text { ITV } \\
(\mathrm{mL})\end{array}$} & \multirow{2}{*}{$\begin{array}{l}\text { PTV } \\
(\mathrm{mL})\end{array}$} & \multirow{2}{*}{$\begin{array}{c}\text { VC pre Hypo-IGRT } \\
(\mathrm{L})\end{array}$} & \multicolumn{2}{|c|}{ FEV1 } & \multirow{2}{*}{$\begin{array}{l}\text { DLCO } \\
\%\end{array}$} & \multirow{2}{*}{$\begin{array}{c}\text { VC post Hypo-IGRT } \\
(\mathrm{L})\end{array}$} & \multicolumn{2}{|c|}{ FEV1 } & \multirow{2}{*}{$\begin{array}{c}\text { DLCO } \\
\%\end{array}$} \\
\hline & & & & $\mathrm{L}$ & $\%$ & & & $L$ & $\%$ & \\
\hline 1 & 128.7 & 255.5 & 2.02 & 1 & 60 & 35 & 1.65 & 0.88 & 53.6 & 27 \\
\hline 2 & 167.4 & 314.28 & 2.66 & 0.84 & 56 & N/M & 2.16 & 0.86 & 28.4 & $\mathrm{~N} / \mathrm{M}$ \\
\hline
\end{tabular}

Hypo-IGRT, hypofractionated image-guided radiotherapy; ITV, internal target volume; PTV, planning target volume; VC, vital capacity; FEV1, forced expiratory volume in 1 second; DLCO, diffusion capacity for carbon monoxide; N/M, not measurable.

hypofractionated radiotherapy in multimorbid patients with early-stage NSCLC without lymph node involvement but not for locally-advance node-positive NSCLC as described in the above-mentioned cases.

A study by He et al. [9] analyzed a subgroup of patients with limited pulmonary function, who received hypofractionated (60 Gy in 20 fractions) CRT via helical tomotherapy and showed good response without an increase in treatment-related toxicity.

Furthermore, previous studies have shown that conventional radiotherapy was associated with significant alterations in patient pulmonary function. Apart from tumor-related morbidity, residual pulmonary function is a major determinant of patient condition and survival. A previous study in locally advanced NSCLC treated with definitive CRT found only 8 months survival in patients with pronounced lung function deficits (VC <60\%, FEV1 <80\% predicted, DLCO <60\%) vs. 16 months in patients with adequate pulmonary function [10]. An analysis from Gopal et al. [11] in 100 lung cancer patients treated with CRT revealed a 1.3\% DLCO decline for each 1\% of total lung volume that received $>20 \mathrm{~Gy}$. Borst et al. [12] also found a significant decrease in the lung function parameters starting at 3 months after definitive CRT.

In the last decade, several groups investigated poor baseline pulmonary function in patients with early-stage NSCLC treated with SBRT and demonstrated that this condition should not be used to exclude patients from treatment [13]. Moderate decline in lung function parameters were revealed 6 to 24 months after SBRT. In addition, Guckenberger et al. [14] reported an improvement of parameters in a subgroup of patients with worse pre-treatment pulmonary function. Another analysis has failed to demonstrate any dose-response relationship for the reduction of function parameters after SBRT.

In conclusion, our case report demonstrates that moderate Hypo-IGRT can be considered in locally advanced nodepositive NSCLC patients with very limited lung function but careful patient selection and monitoring as well as experience with SBRT technique are important prerequisites. Prospective studies for the evaluation of safety and efficacy of this approach are urgently necessary; we are currently planning a prospective study pending approval from the ethics committee of the Ludwig-Maximilians University of Munich.

\section{Conflict of Interest}

No potential conflict of interest relevant to this article was reported.

\section{Acknowledgments}

The Authors acknowledge Josephine Rauch, MD and Maximilian Niyazi, MD, PhD who participated in the writing and technical editing of the manuscript.

\section{References}

1. Walasek T, Sas-Korczynska B, Dąbrowski T, et al. Palliative thoracic radiotherapy for patients with advanced non-small cell lung cancer and poor performance status. Lung Cancer 2015;87:130-5.

2. Shultz DB, Diehn M, Loo BW Jr. To SABR or not to SABR? Indications and contraindications for stereotactic ablative radiotherapy in the treatment of early-stage, oligometastatic, or oligoprogressive non-small cell lung cancer. Semin Radiat Oncol 2015;25:78-86.

3. Amini A, Lin SH, Wei C, Allen P, Cox JD, Komaki R. Accelerated hypofractionated radiation therapy compared to conventionally fractionated radiation therapy for the treatment of inoperable non-small cell lung cancer. Radiat Oncol 2012;7:33.

4. Nguyen LN, Komaki R, Allen P, Schea RA, Milas L. Effectiveness of accelerated radiotherapy for patients with inoperable nonsmall cell lung cancer (NSCLC) and borderline prognostic factors without distant metastasis: a retrospective review. Int J Radiat Oncol Biol Phys 1999;44:1053-6.

5. Kim TH, Cho KH, Pyo HR, et al. Dose-volumetric parameters 
for predicting severe radiation pneumonitis after threedimensional conformal radiation therapy for lung cancer. Radiology 2005;235:208-15.

6. Hernando ML, Marks LB, Bentel GC, et al. Radiation-induced pulmonary toxicity: a dose-volume histogram analysis in 201 patients with lung cancer. Int J Radiat Oncol Biol Phys 2001;51:650-9.

7. Graham MV, Purdy JA, Emami B, et al. Clinical dose-volume histogram analysis for pneumonitis after 3D treatment for non-small cell lung cancer (NSCLC). Int J Radiat Oncol Biol Phys 1999:45:323-9.

8. Chiang A, Thibault I, Warner A, et al. A comparison between accelerated hypofractionation and stereotactic ablative radiotherapy (SABR) for early-stage non-small cell lung cancer (NSCLC): Results of a propensity score-matched analysis. Radiother Oncol 2016;118:478-84.

9. He J, Huang Y, Chen $Y$, et al. Feasibility and efficacy of helical intensity-modulated radiotherapy for stage III non-small cell lung cancer in comparison with conventionally fractionated 3D-CRT. J Thorac Dis 2016;8:862-71.

10. Semrau S, Klautke G, Fietkau R. Baseline cardiopulmonary function as an independent prognostic factor for survival of inoperable non-small-cell lung cancer after concurrent chemoradiotherapy: a single-center analysis of 161 cases. Int J Radiat Oncol Biol Phys 2011;79:96-104.

11. Gopal R, Starkschall G, Tucker SL, et al. Effects of radiotherapy and chemotherapy on lung function in patients with non-small-cell lung cancer. Int J Radiat Oncol Biol Phys 2003;56:114-20.

12. Borst GR, De Jaeger K, Belderbos JS, Burgers SA, Lebesque JV. Pulmonary function changes after radiotherapy in non-smallcell lung cancer patients with long-term disease-free survival. Int J Radiat Oncol Biol Phys 2005;62:639-44.

13. Guckenberger $M$, Kestin $L L$, Hope $A J$, et al. Is there a lower limit of pretreatment pulmonary function for safe and effective stereotactic body radiotherapy for early-stage non-small cell lung cancer? J Thorac Oncol 2012;7:542-51.

14. Guckenberger M, Klement RJ, Kestin LL, et al. Lack of a doseeffect relationship for pulmonary function changes after stereotactic body radiation therapy for early-stage non-small cell lung cancer. Int J Radiat Oncol Biol Phys 2013;85:1074-81. 